\title{
Estimating the risk of acute rheumatic fever in New Zealand by age, ethnicity and deprivation
}

\author{
J. K. GURNEY ${ }^{1}$, J. STANLEY ${ }^{1}$, M. G. BAKER ${ }^{1}$, N. J. WILSON ${ }^{2}$ AND \\ D. SARFATI ${ }^{1}$ \\ ${ }^{1}$ Department of Public Health, University of Otago, Wellington, New Zealand \\ ${ }^{2}$ Starship Children's Hospital, Auckland District Health Board, Auckland, New Zealand
}

Received 29 January 2016; Final revision 30 May 2016; Accepted 2 June 2016; first published online 17 June 2016

\section{SUMMARY}

In New Zealand, efforts to control acute rheumatic fever (ARF) and its sequelae have focused on school-age children in the poorest socioeconomic areas; however, it is unclear whether this approach is optimal given the strong association with demographic risk factors other than deprivation, especially ethnicity. The aim of this study was to estimate the stratum-specific risk of ARF by key sociodemographic characteristics. We used hospitalization and disease notification data to identify new cases of ARF between 2010 and 2013, and used population count data from the 2013 New Zealand Census as our denominator. Poisson logistic regression methods were used to estimate stratum-specific risk of ARF development. The likelihood of ARF development varied considerably by age, ethnicity and deprivation strata: while risk was greatest in Māori and Pacific children aged 10-14 years residing in the most extreme deprivation, both of these ethnic groups experienced elevated risk across a wide age range and across deprivation levels. Interventions that target populations based on deprivation will include the highest-risk strata, but they will also (a) include groups with very low risk of ARF, such as non-Māori/non-Pacific children; and $(b)$ exclude groups with moderate risk of ARF, such as Māori and Pacific individuals living outside high deprivation areas.

Key words: Public health, rheumatic fever, rheumatic heart disease.

\section{INTRODUCTION}

Acute rheumatic fever (ARF) is an autoimmune disorder resulting from exposure to strains of group A Streptococcus (GAS) bacterium. Typical features of ARF include inflammation of joints, the central nervous system and, most seriously, cardiac valve tissue - with more than half of all ARF cases expected to develop rheumatic heart disease (RHD) [1].

\footnotetext{
* Author for correspondence: Dr J. K. Gurney, Department of Public Health, University of Otago, Wellington, PO Box 7343, Wellington, New Zealand.

(Email: jason.gurney@otago.ac.nz)
}

Despite evidence linking GAS infection to ARF development, the aetiology of the latter remains poorly understood. Typing of GAS isolates obtained from New Zealand ARF cases from 2006 to 2014 identified few of the so-called rheumatogenic emm types, but found a high proportion of types previously associated with pyoderma - supporting a potential role for skin infection in ARF [2]. GAS carriage is relatively common, with $12 \%$ of all children carrying the bacteria asymptomatically in their throat [3]. The fact that only a small proportion of GAS infections lead to ARF suggests that there are other factors in addition to GAS infection itself that have a strong modifying effect on host susceptibility to ARF development [4]. 
ARF is a disease of childhood, with most cases occurring in those aged 4-19 years [5, 6]. There is a strong deprivation gradient for this disease, which is thought to be driven by heightened exposure to factors such as dampness in the home and sore throat frequency [7]. In New Zealand, ARF is now almost entirely restricted to Māori and Pacific peoples $[5,6]$ - with these populations 20-40 times more likely to be diagnosed with ARF than the European population $[5,6,8]$.

Efforts to control ARF and its sequelae, such as throat swabbing programmes and screening for asymptomatic RHD, have to date focused on schools in the lowest socioeconomic areas [9]. This targeting was based on the strong association between deprivation and ARF incidence; however, it is not clear whether this approach is the optimal one given the strong association with socio-demographic risk factors other than deprivation, especially ethnicity [5].

The aim of this paper is to estimate the stratumspecific risk of ARF in the New Zealand contextallowing us to simultaneously combine the effects of known socio-demographic risk factors (i.e. age, ethnicity, deprivation). Stratum-specific analyses allow us to assess the strength of associations between exposure to these risk factors and disease incidence. In addition, we aimed to assess the potential for targeting of interventions based on quantifying the number of individuals belonging to the highest-risk groups, both for the total New Zealand population and also by geographical region.

\section{METHODS}

\section{Participants - cases}

Each new case of ARF identified over the 4-year period 2010-2013 was included in this study. Diagnosis of ARF in New Zealand is made according to a modified version of the Jones Criteria [10]. To identify cases, we requested hospitalization data (National Minimum Dataset; NMDS) from the Ministry of Health pertaining to all hospitalizations with an admission date that occurred between 2010 and 2013 in which a primary diagnosis of ARF was made (ICD-10-AM codes: I00-I02). Second, we requested notification data ('EpiSurv') from the Institute of Environmental Science and Research (ESR) for all new cases of ARF reported during 2010-2013. The notification data were then merged (by unique patient identifier) with the hospitalization data. In the event that a patient and associated data existed in both cohorts, a 'give-way' rule was applied whereby hospitalization data were preferred to notification data (as notifications can be delayed).

For any given patient, only data pertaining to the earliest ARF diagnosis between 2010 and 2013 were retained since the aim was to identify incident cases. Since it was feasible that the diagnosis dates recorded in the hospitalization and notification datasets might not be identical, a 4-week diagnosis 'window' was employed when merging these datasets - whereby hospitalization and notification data were assumed to refer to the same underlying ARF event provided the diagnosis dates were within 4 weeks of each other. The earliest recorded date of diagnosis for a given ARF case was retained as the index date for that patient.

After merging the hospitalization and notification datasets, a total of 929 unique ARF cases were identified. Since the aim was to identify incident cases of ARF rather than recurrences, we excluded those who had a recorded history of ARF (prior to 2010) or RHD (any time prior to the ARF diagnosis date) using a case-identification algorithm. This is the same case identification algorithm that is used by the New Zealand Ministry of Health to determine ARF incidence based on hospitalization data. The algorithm restricts the attribution of ARF incidence to those patients for whom: (1) ARF is the principal diagnosis only; (2) no previous principal or additional diagnoses of ARF are recorded; and (3) no previous principal or additional diagnoses of RHD are recorded [11]. In order to apply this algorithm, we linked all patients in the cohort to their hospitalization records from 1988 [the year the National Health Index (NHI) became universal] [12] or their birth (if born since 1988), for evidence of previous ARF (ICD-10-AM codes: I00-I02) or RHD (I05-I09). Based on this algorithm, we excluded 156 patients from further analysis. Using hospitalization data, we also excluded those who were recorded as being a non-New Zealand resident at the time of their ARF $(n=38)$. Following exclusions, a final cohort $(n=$ 733) remained for further analysis. Of this total, 495 cases ( $68 \%$ of final cohort) were recorded in both the hospitalization and notification datasets, $176(24 \%)$ were only recorded in hospitalization data, and 62 $(8 \%)$ were only recorded in notification data. These cases are the same as those used in a previous study outlining the incidence of ARF in New Zealand between 2010 and 2013 [8]. 


\section{Participants - source population}

The 2013 New Zealand Census population served as the source population for this study. We sought and received population count data, stratified by age, ethnicity, deprivation, and geographical location, from the developers of the NZDep 2013 tool [13]. The source population included all individuals belonging to one of the prioritized ethnicity groups (Māori, Pacific, Asian, non-Māori/non-Pacific/non-Asian; see 'Variables and final dataset' section below), stratified by age group and deprivation strata.

\section{Variables and final dataset}

For ARF cases, ethnicity, geographical location [domicile code or Census Area Unit (CAU)] and date of birth/age were determined from both the hospitalization and notification datasets, with hospitalization data preferred to notification data in line with our 'give-way' rule. If hospitalization data were incomplete for these variables, then notification data (where available) were used to fill these gaps.

For the source population, unit-level records (i.e. one row per individual) were created based on the age-, ethnicity- and deprivation-stratified population count data. For example, 39768 individuals from the source population were aged $0-4$ years, of 'European/other' ethnicity, and had an NZDep score of 1-2; thus, our final dataset included 39768 records for this combination of factors. Our variables are further described below.

- Ethnicity. For both ARF cases and the source population, ethnicity was determined using the prioritized ethnicity approach [5, 6], whereby if more than one ethnic group is nominated by an individual, one ethnicity is attributed based on a prioritization algorithm [14]. Using this approach, patients were placed into either Māori, Pacific or Asian ethnic groups (prioritized in that order), while those who were not recorded as having any of these three affiliations were recorded as non-Māori/non-Pacific/non-Asian (described as 'European/other' in this paper).

- Age. For ARF cases, patient age was determined from date of birth (NMDS) or age at diagnosis (EpiSurv) data. Age group in the source population is available as categorized counts, and so for both ARF cases and the source population age was treated as a categorical variable (age groups: $0-4,5-9$, $10-14,15-19,20-24,25-29, \geqslant 30$ years).
- Deprivation. For both ARF cases and the source population, deprivation was determined using quintiles of the NZDep 2013 index, which attributes a level of deprivation based on the CAU where the patient resided at the time of ARF diagnosis. Incomplete CAU data $(n=2,0 \cdot 3 \%$ of cohort) and a lack of NZDep score availability $(n=20,2 \cdot 7 \%$ of cohort) prevented attribution of NZDep in 22 (3\%) ARF cases.

- Geographical location. The geographical location (District Health Board) of each case was attributed based on the CAU where they lived at the time of either (a) ARF incidence (for ARF cases) or (b) time of the 2013 Census (for the source population). In urban areas, the boundaries of a CAU generally coincide with a suburb, while CAUs in rural areas generally span larger geographical areas [15]. For ARF cases, CAU was mapped across from the domicile code (another geographical unit based on the address where the individual is usually resident) attributed to the patient at time of hospitalization. Notification (EpiSurv) data (if available) were used to augment CAU data in those cases where (a) the domicile code mapped to a non-existent or out-of-date CAU or $(b)$ the domicile code was absent from the hospitalization data. Following augmentation, CAU could not be determined for a total of two $(0 \cdot 3 \%)$ ARF cases. Using a concordance file [16], CAU was then used to determine the District Health Board that a patient was residing in at the time of their diagnosis. District Health Board could not be determined for a total of $10(1 \cdot 4 \%)$ ARF cases due to missing concordance data.

The final dataset included complete data for $711 \mathrm{ARF}$ cases and 4227981 individuals from the source population (Fig. 1). Each individual was attributed a unique study identifier, and merged into a final cohort with 4228692 individual records.

\section{Statistical analysis}

To estimate stratum-specific rates of ARF, we ran Poisson regression models that included our categorical predictor variables (age, ethnicity, deprivation quintile). For each variable, a reference category was selected (age: $\geqslant 30$ years; ethnicity: European/other; deprivation: NZDep deciles 1-2). Predicted rates of ARF for each stratum were calculated from the Poisson models based on the independent impact of 


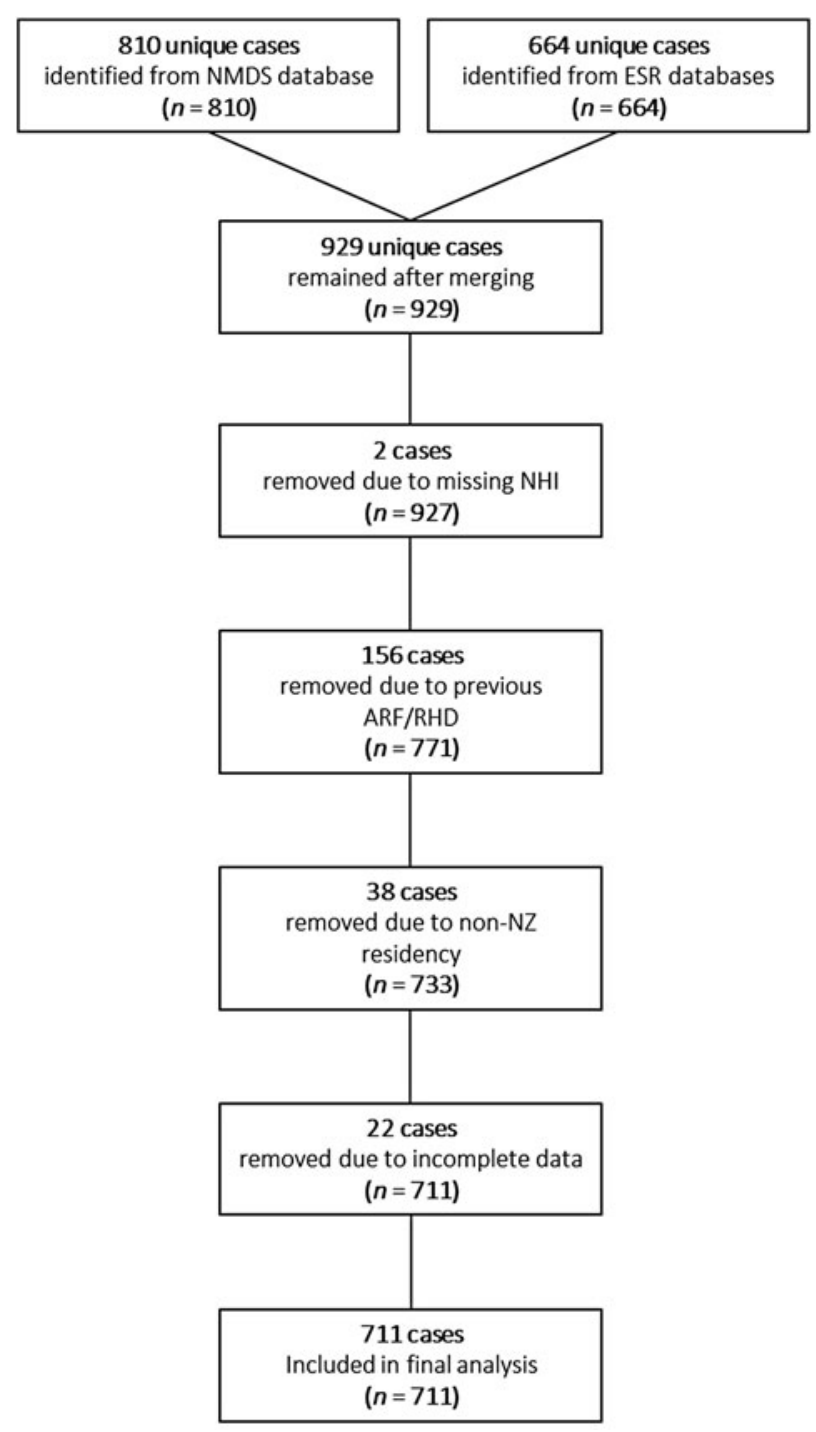

Fig. 1. Acute rheumatic fever (ARF) case inclusion/ exclusion flowchart. NMDS, National Minimum Dataset; ESR, Institute of Environmental Science and Research; NHI, National Health Index; RHD, rheumatic heart disease.

age, ethnicity, and deprivation, by solving the model for each stratum (i.e. summing the appropriate beta coefficients for each stratum). Adjusted rate ratios (aRRs) were derived from the model, along with corresponding 95\% confidence intervals (CIs). Rates of ARF for each stratum were also derived from the Poisson models, and expressed in terms of personyears at risk (pyar). For simplicity, the contribution of each individual record to the person-time denominator was fixed at 4 person-years (i.e. 2010-2013).

All analyses were performed in SAS v. 9.3 (SAS Institute Inc., USA) and Microsoft Excel (Microsoft Corp., USA).
Table 1. Demographic characteristics of the source population and ARF cases diagnosed between 2010 and 2013

\begin{tabular}{|c|c|c|c|c|}
\hline & \multicolumn{2}{|c|}{$\begin{array}{l}\text { Source } \\
\text { population, 2013* }\end{array}$} & \multicolumn{2}{|c|}{$\begin{array}{l}\text { ARF cases, } \\
\text { 2010-2013 }\end{array}$} \\
\hline & $N$ & $(\%)$ & $n$ & $(\%)$ \\
\hline Total & 4227981 & $(100)$ & 711 & $(100)$ \\
\hline \multicolumn{5}{|l|}{ Sex } \\
\hline Female & 2168916 & $(51 \cdot 3)$ & 312 & $(43 \cdot 9)$ \\
\hline Male & 2059125 & $(48 \cdot 7)$ & 399 & $(56 \cdot 1)$ \\
\hline \multicolumn{5}{|l|}{ Age,years } \\
\hline$<5$ & 291960 & $(6 \cdot 9)$ & 3 & $(0 \cdot 4)$ \\
\hline $5-9$ & 286698 & $(6 \cdot 8)$ & 179 & $(25 \cdot 2)$ \\
\hline $10-14$ & 286725 & $(6 \cdot 8)$ & 301 & $(42 \cdot 3)$ \\
\hline $15-19$ & 295659 & (7) & 101 & $(14 \cdot 2)$ \\
\hline $20-24$ & 290589 & $(6 \cdot 9)$ & 59 & $(8 \cdot 3)$ \\
\hline $25-29$ & 258024 & $(6 \cdot 1)$ & 26 & $(3 \cdot 7)$ \\
\hline$\geqslant 30$ & 2518326 & $(59 \cdot 6)$ & 42 & $(5 \cdot 9)$ \\
\hline \multicolumn{5}{|l|}{ Ethnicity } \\
\hline European/other & 2928717 & $(69 \cdot 3)$ & 48 & $(6 \cdot 8)$ \\
\hline Māori & 598248 & $(14 \cdot 1)$ & 387 & $(54 \cdot 4)$ \\
\hline Pacific & 246747 & $(5 \cdot 8)$ & 267 & $(37 \cdot 6)$ \\
\hline Asian & 454269 & $(10 \cdot 7)$ & 9 & $(1 \cdot 3)$ \\
\hline \multicolumn{5}{|l|}{ Deprivation $\dagger$} \\
\hline 1-2 (least deprived) & 873312 & $(20 \cdot 7)$ & 13 & $(1 \cdot 8)$ \\
\hline $3-4$ & 853623 & $(20 \cdot 2)$ & 36 & $(5 \cdot 1)$ \\
\hline $5-6$ & 837933 & $(19 \cdot 8)$ & 53 & $(7 \cdot 5)$ \\
\hline $7-8$ & 829992 & $(19 \cdot 6)$ & 109 & $(15 \cdot 3)$ \\
\hline 9-10 (most deprived) & 833121 & $(19 \cdot 7)$ & 500 & $(70 \cdot 3)$ \\
\hline
\end{tabular}

ARF, Acute rheumatic fever.

* The source population included individuals from the 2013 New Zealand Census belonging to each ethnic group (Māori, Pacific, Asian, non-Māori/non-Pacific/non-Asian), stratified by age and deprivation stratum.

$\dagger$ Defined using 2013 NZDep deciles.

\section{RESULTS}

Demographic characteristics of the total cohort are given in Table 1. We observed that fewer ARF cases were female than male $(44 \%$ vs. $56 \%)$. Congruent with expectations, the majority ( $82 \%$ ) of ARF cases were aged between 5 and 19 years, were Māori or Pacific (92\%), and resided in NZDep deciles 9-10 (70\%).

Adjusted rate ratios from our Poisson regression model are given in Table 2. Those aged 10-14 years were $>30$ times more likely to develop ARF compared to those aged $\geqslant 30$ years, adjusting for differences in ethnicity and deprivation (aRR 31.9, 95\% CI $23 \cdot 0$ 44.0]; while those aged 5-9 years were 18 times more likely (aRR $18 \cdot 5,95 \%$ CI 13.2-25.9) to develop ARF. Māori and Pacific peoples were 14-21 times 
Table 2. Adjusted rate ratios and confidence intervals of acute rheumatic fever from Poisson regression modelling

\begin{tabular}{lll}
\hline \hline & aRR & $(95 \% \mathrm{CI})$ \\
\hline Age, years & & \\
$\quad 0-4$ & $0 \cdot 3$ & $(0 \cdot 09-0 \cdot 94)$ \\
$5-9$ & $18 \cdot 45$ & $(13 \cdot 17-25 \cdot 85)$ \\
$10-14$ & $31 \cdot 86$ & $(23 \cdot 03-44 \cdot 05)$ \\
$15-19$ & $11 \cdot 19$ & $(7 \cdot 8-16 \cdot 05)$ \\
$20-24$ & $7 \cdot 46$ & $(5 \cdot 02-11 \cdot 09)$ \\
$25-29$ & $4 \cdot 22$ & $(2 \cdot 59-6 \cdot 89)$ \\
20 & Reference & \\
Ethnicity & & \\
European/other & Reference & \\
Māori & $14 \cdot 46$ & $(10 \cdot 62-19 \cdot 71)$ \\
Pacific & $20 \cdot 58$ & $(14 \cdot 93-28 \cdot 36)$ \\
Asian & $0 \cdot 89$ & $(0 \cdot 44-1 \cdot 81)$ \\
Deprivation (NZDep quintile) & & \\
$1-2$ (least deprived) & Reference & \\
3-4 & $2 \cdot 33$ & $(1 \cdot 23-4 \cdot 39)$ \\
5-6 & $2 \cdot 68$ & $(1 \cdot 46-4 \cdot 92)$ \\
$7-8$ & $3 \cdot 89$ & $(2 \cdot 18-6 \cdot 95)$ \\
9-10 (most deprived) & $8 \cdot 96$ & $(5 \cdot 12-15 \cdot 66)$ \\
\hline \hline
\end{tabular}

aRR, Adjusted rate ratio; CI, confidence interval.

more likely to develop the disease compared to European/other individuals after adjusting for differences in age and deprivation (Māori: aRR 14.5, 95\% CI 10·6-19.7; Pacific: aRR 20.6, 95\% CI 14.9-28.4), while those residing in areas with NZDep deciles 910 were nearly nine times more likely to develop ARF compared to NZDep deciles 1-2 after adjusting for differences in age and ethnicity (aRR 8.9, 95\% CI $5 \cdot 1-15 \cdot 7)$.

Stratum-specific rates of ARF are presented in Table 3. The European/other and Asian cohorts both had minimal risk of ARF, regardless of age and deprivation strata. The Māori cohort had rates $>1$ case/1000 pyar for multiple age and deprivation strata, with the highest occurring in 10- to 14-year-olds residing in NZDep deciles 9-10 (4.4 cases 1000 pyar). The Pacific cohort showed a nearidentical pattern of stratum-specific rates, with peak risk also occurring in 10- to 14-year-olds residing in NZDep deciles 9-10 (6.2 cases/1000 pyar).

The absolute number of individuals in each of the risk strata is presented in Table 4 for the ethnicitystratified New Zealand population, and by District Health Board in Supplementary Table S2). Those strata with a rate of disease $\geqslant 1$ case $/ 1000$ pyar (from Table 3) are highlighted in each table. For example, the total number of Māori children in New Zealand aged 10-14 years and residing in NZDep deciles 9-10 was 25833; while the total number of Pacific children belonging to these strata was 14748 .

\section{DISCUSSION}

The aim of this study was to simultaneously combine the effects of known socio-demographic predictors of ARF incidence in New Zealand, in order to estimate stratum-specific rates of disease. Using this information, we were also able to quantify the number of individuals belonging to the highest-risk population groups - both for the total New Zealand population (Table 4) and also by geographical region (Supplementary Table S2).

In line with expectations, we observed that the European/other and Asian populations have minimal risk of ARF development, with Māori and Pacific peoples carrying the most significant burden of disease. (While our observations are similar to those reported previously $[5,6]$, it is worth noting that they are not directly comparable since they are adjusted for different demographic variables.) Those at greatest risk of ARF in New Zealand are Māori and Pacific children, aged 5-14 years, residing in the most deprived areas of the country (NZDep 9-10). However, we did observe elevated risk of ARF in Mãori and Pacific peoples outside of these highest-risk strata - with patterns of elevated risk nearly identical between Māori and Pacific peoples (Table 3).

It is clear that age, ethnicity and deprivation all contribute independently to ARF risk. Age is obviously a crucial risk factor in the development of ARF, with most cases occurring in childhood [6]. However, the association between the other two risk factors and ARF risk is more complex: for instance, if deprivation were the only important risk factor for ARF, then we would observe similarly elevated rates of disease in all ethnic groups residing in NZDep deciles 9-10; and if Māori or Pacific ethnicity were the only important risk factor, then we would expect to see similarly elevated rates of disease across deprivation strata within these ethnic groups. The data presented here (and elsewhere) clearly indicate that the risk of ARF is modified by a complex combination of (and interaction between) these risk factors.

In order to understand more about the aetiology of ARF based on the socio-demographic predictors used in this study, we need to understand more about what they represent. Since ARF is an immune-mediated disease-rather than an infectious disease per se-the host response to GAS infection is particularly 
Table 3. Stratum-specific rate of acute rheumatic fever (per 1000 pyar), by age, ethnicity and deprivation strata

\begin{tabular}{|c|c|c|c|c|c|c|c|c|c|c|}
\hline \multirow[b]{3}{*}{ Age group, yr } & \multicolumn{10}{|c|}{ Deprivation (NZDep quintile) } \\
\hline & \multicolumn{2}{|c|}{ 1-2 (least deprived) } & \multicolumn{2}{|l|}{$3-4$} & \multicolumn{2}{|l|}{$5-6$} & \multicolumn{2}{|l|}{$7-8$} & \multicolumn{2}{|c|}{ 9-10 (most deprived) } \\
\hline & Rate* & $(95 \% \mathrm{CI})$ & Rate* & $(95 \% \mathrm{CI})$ & Rate* & $(95 \% \mathrm{CI})$ & Rate* & $(95 \% \mathrm{CI})$ & Rate* & $(95 \% \mathrm{CI})$ \\
\hline \multicolumn{11}{|l|}{ European/other } \\
\hline $0-4$ & 0 & $(0-0)$ & 0 & $(0-0)$ & 0 & $(0-0)$ & 0 & $(0-0)$ & 0 & $(0-0)$ \\
\hline $5-9$ & 0 & $(0-0)$ & 0 & $(0-0 \cdot 1)$ & $0 \cdot 1$ & $(0-0 \cdot 1)$ & $0 \cdot 1$ & $(0 \cdot 1-0 \cdot 1)$ & $0 \cdot 2$ & $(0 \cdot 1-0 \cdot 2)$ \\
\hline $10-14$ & 0 & $(0-0 \cdot 1)$ & $0 \cdot 1$ & $(0 \cdot 1-0 \cdot 1)$ & $0 \cdot 1$ & $(0 \cdot 1-0 \cdot 1)$ & $0 \cdot 1$ & $(0 \cdot 1-0 \cdot 2)$ & $0 \cdot 3$ & $(0 \cdot 2-0 \cdot 4)$ \\
\hline $15-19$ & 0 & $(0-0)$ & 0 & $(0-0)$ & 0 & $(0-0)$ & 0 & $(0-0 \cdot 1)$ & $0 \cdot 1$ & $(0 \cdot 1-0 \cdot 2)$ \\
\hline $20-24$ & 0 & $(0-0)$ & 0 & $(0-0)$ & 0 & $(0-0)$ & 0 & $(0-0)$ & $0 \cdot 1$ & $(0-0 \cdot 1)$ \\
\hline $25-29$ & 0 & $(0-0)$ & 0 & $(0-0)$ & 0 & $(0-0)$ & 0 & $(0-0)$ & 0 & $(0-0 \cdot 1)$ \\
\hline$\geqslant 30$ & 0 & $(0-0)$ & 0 & $(0-0)$ & 0 & $(0-0)$ & 0 & $(0-0)$ & 0 & $(0-0)$ \\
\hline \multicolumn{11}{|l|}{ Māori } \\
\hline $0-4$ & 0 & $(0-0)$ & 0 & $(0-0)$ & 0 & $(0-0)$ & 0 & $(0-0 \cdot 1)$ & 0 & $(0-0 \cdot 1)$ \\
\hline $5-9$ & $0 \cdot 3$ & $(0 \cdot 2-0 \cdot 5)$ & $0 \cdot 7$ & $(0 \cdot 5-0 \cdot 9)$ & $0 \cdot 8$ & $(0 \cdot 6-1)$ & $1 \cdot 1$ & $(0 \cdot 9-1 \cdot 4)$ & $2 \cdot 5$ & $(2 \cdot 1-3)$ \\
\hline $10-14$ & $0 \cdot 5$ & $(0 \cdot 3-0 \cdot 9)$ & $1 \cdot 1$ & $(0 \cdot 8-1 \cdot 6)$ & $1 \cdot 3$ & $(1-1 \cdot 8)$ & 1.9 & $(1 \cdot 5-2 \cdot 4)$ & $4 \cdot 4$ & $(3 \cdot 8-5 \cdot 1)$ \\
\hline 15-19 & $0 \cdot 2$ & $(0 \cdot 1-0 \cdot 3)$ & $0 \cdot 4$ & $(0 \cdot 3-0 \cdot 6)$ & $0 \cdot 5$ & $(0 \cdot 3-0 \cdot 6)$ & $0 \cdot 7$ & $(0 \cdot 5-0 \cdot 9)$ & $1 \cdot 5$ & $(1 \cdot 2-1 \cdot 9)$ \\
\hline $20-24$ & $0 \cdot 1$ & $(0 \cdot 1-0 \cdot 2)$ & $0 \cdot 3$ & $(0 \cdot 2-0 \cdot 4)$ & $0 \cdot 3$ & $(0 \cdot 2-0 \cdot 4)$ & $0 \cdot 4$ & $(0 \cdot 3-0 \cdot 6)$ & 1 & $(0 \cdot 8-1 \cdot 3)$ \\
\hline $25-29$ & $0 \cdot 1$ & $(0-0 \cdot 1)$ & $0 \cdot 2$ & $(0 \cdot 1-0 \cdot 3)$ & $0 \cdot 2$ & $(0 \cdot 1-0 \cdot 3)$ & $0 \cdot 3$ & $(0 \cdot 2-0 \cdot 4)$ & $0 \cdot 6$ & $(0 \cdot 4-0 \cdot 9)$ \\
\hline$\geqslant 30$ & 0 & $(0-0)$ & 0 & $(0-0 \cdot 1)$ & 0 & $(0-0 \cdot 1)$ & $0 \cdot 1$ & $(0-0 \cdot 1)$ & $0 \cdot 1$ & $(0 \cdot 1-0 \cdot 2)$ \\
\hline \multicolumn{11}{|l|}{ Pacific } \\
\hline $0-4$ & 0 & $(0-0)$ & 0 & $(0-0)$ & 0 & $(0-0 \cdot 1)$ & 0 & $(0-0 \cdot 1)$ & $0 \cdot 1$ & $(0-0 \cdot 2)$ \\
\hline $5-9$ & $0 \cdot 4$ & $(0 \cdot 2-0 \cdot 7)$ & $0 \cdot 9$ & $(0 \cdot 6-1 \cdot 4)$ & $1 \cdot 1$ & $(0 \cdot 8-1 \cdot 5)$ & $1 \cdot 6$ & $(1 \cdot 2-2)$ & $3 \cdot 6$ & $(3-4 \cdot 3)$ \\
\hline $10-14$ & $0 \cdot 7$ & $(0 \cdot 4-1 \cdot 2)$ & $1 \cdot 6$ & $(1 \cdot 1-2 \cdot 3)$ & $1 \cdot 9$ & $(1 \cdot 4-2 \cdot 5)$ & $2 \cdot 7$ & $(2 \cdot 1-3 \cdot 4)$ & $6 \cdot 2$ & $(5 \cdot 4-7 \cdot 3)$ \\
\hline 15-19 & $0 \cdot 2$ & $(0 \cdot 1-0 \cdot 4)$ & $0 \cdot 6$ & $(0 \cdot 4-0 \cdot 8)$ & $0 \cdot 7$ & $(0 \cdot 5-0 \cdot 9)$ & 1 & $(0 \cdot 7-1 \cdot 3)$ & $2 \cdot 2$ & $(1 \cdot 8-2 \cdot 7)$ \\
\hline $20-24$ & $0 \cdot 2$ & $(0 \cdot 1-0 \cdot 3)$ & $0 \cdot 4$ & $(0 \cdot 2-0 \cdot 6)$ & $0 \cdot 4$ & $(0 \cdot 3-0 \cdot 6)$ & $0 \cdot 6$ & $(0 \cdot 5-0 \cdot 9)$ & $1 \cdot 5$ & $(1 \cdot 1-1 \cdot 9)$ \\
\hline $25-29$ & $0 \cdot 1$ & $(0-0 \cdot 2)$ & $0 \cdot 2$ & $(0 \cdot 1-0 \cdot 4)$ & $0 \cdot 2$ & $(0 \cdot 2-0 \cdot 4)$ & $0 \cdot 4$ & $(0 \cdot 2-0 \cdot 6)$ & $0 \cdot 8$ & $(0 \cdot 6-1 \cdot 2)$ \\
\hline$\geqslant 30$ & 0 & $(0-0)$ & $0 \cdot 1$ & $(0-0 \cdot 1)$ & $0 \cdot 1$ & $(0-0 \cdot 1)$ & $0 \cdot 1$ & $(0 \cdot 1-0 \cdot 1)$ & $0 \cdot 2$ & $(0 \cdot 1-0 \cdot 3)$ \\
\hline \multicolumn{11}{|l|}{ Asian } \\
\hline $0-4$ & 0 & $(0-0)$ & 0 & $(0-0)$ & 0 & $(0-0)$ & 0 & $(0-0)$ & 0 & $(0-0)$ \\
\hline $5-9$ & 0 & $(0-0)$ & 0 & $(0-0 \cdot 1)$ & 0 & $(0-0 \cdot 1)$ & $0 \cdot 1$ & $(0-0 \cdot 1)$ & $0 \cdot 2$ & $(0 \cdot 1-0 \cdot 3)$ \\
\hline $10-14$ & 0 & $(0-0 \cdot 1)$ & $0 \cdot 1$ & $(0-0 \cdot 1)$ & $0 \cdot 1$ & $(0-0 \cdot 2)$ & $0 \cdot 1$ & $(0 \cdot 1-0 \cdot 2)$ & $0 \cdot 3$ & $(0 \cdot 1-0 \cdot 5)$ \\
\hline $15-19$ & 0 & $(0-0)$ & 0 & $(0-0 \cdot 1)$ & 0 & $(0-0 \cdot 1)$ & 0 & $(0-0 \cdot 1)$ & $0 \cdot 1$ & $(0-0 \cdot 2)$ \\
\hline $20-24$ & 0 & $(0-0)$ & 0 & $(0-0)$ & 0 & $(0-0)$ & 0 & $(0-0 \cdot 1)$ & $0 \cdot 1$ & $(0-0 \cdot 1)$ \\
\hline $25-29$ & 0 & $(0-0)$ & 0 & $(0-0)$ & 0 & $(0-0)$ & 0 & $(0-0)$ & 0 & $(0-0 \cdot 1)$ \\
\hline$\geqslant 30$ & 0 & $(0-0)$ & 0 & $(0-0)$ & 0 & $(0-0)$ & 0 & $(0-0)$ & 0 & $(0-0)$ \\
\hline
\end{tabular}

CI, Confidence interval; pyar, person-years at risk.

* Rate per 1000 pyar. Areas in grey indicate stratum-specific predicted rate of 1 case/1000 pyar.

important. Age appears to be a profoundly important host factor, since ARF is rare in children aged $<5$ years and almost unknown in children aged $<3$ years suggesting that the aetiology of rheumatic fever requires a maturing immune system. The elevated risk for Māori and Pacific peoples of this disease is so markedly large compared to other infectious diseases [17] that this vulnerability would suggest a genetic contribution (the heritability of ARF, based on twin studies, is estimated at about 60\%) [18]. However, an argument against the strength of this genetic contribution is the fact that ARF once had high incidence in European populations - both in New Zealand and elsewhere.
Finally, the independent association with deprivation provides strong evidence that exposures associated with poverty are important, and these are likely to be mediated through increased rates of GAS or some other co-factors that we are unaware of.

\section{Implications for prevention and screening interventions}

As discussed in the Introduction, efforts to date to control ARF and its sequelae have focused on schools in the lowest socioeconomic areas - a logical strategy, given $(a)$ the known high incidence of this disease in children, and $(b)$ the known link between deprivation and ARF development. 
Table 4. 2013 New Zealand Census ethnicity-stratified population counts*, by age and deprivation strata

\begin{tabular}{|c|c|c|c|c|c|}
\hline \multirow[b]{2}{*}{ Age group, yr } & \multicolumn{5}{|c|}{ Deprivation (NZDep quintile) } \\
\hline & $\begin{array}{l}1-2 \\
\text { (least deprived) }\end{array}$ & $3-4$ & $5-6$ & $7-8$ & $\begin{array}{l}9-10 \\
\text { (most deprived) }\end{array}$ \\
\hline \multicolumn{6}{|c|}{ European/other } \\
\hline $0-4$ & 39768 & 37107 & 34161 & 29151 & 21837 \\
\hline $5-9$ & 48828 & 39066 & 33156 & 26301 & 18906 \\
\hline $10-14$ & 50565 & 40185 & 33444 & 26202 & 18732 \\
\hline $15-19$ & 45621 & 38106 & 34179 & 33375 & 25911 \\
\hline $20-24$ & 32874 & 35253 & 38826 & 39408 & 29319 \\
\hline $25-29$ & 27354 & 34488 & 36933 & 34005 & 23046 \\
\hline$\geqslant 30$ & 485241 & 443781 & 403092 & 352500 & 237996 \\
\hline \multicolumn{6}{|l|}{ Māori } \\
\hline $0-4$ & 5586 & 7857 & 11469 & 16236 & 29904 \\
\hline $5-9$ & 5973 & 7704 & 10785 & 14985 & 27678 \\
\hline $10-14$ & 5961 & 7623 & 10305 & 14358 & 25833 \\
\hline 15-19 & 5139 & 6675 & 9372 & 13692 & 23724 \\
\hline $20-24$ & 3681 & 5250 & 8064 & 11727 & 19647 \\
\hline $25-29$ & 2475 & 4197 & 6216 & 9141 & 15039 \\
\hline$\geqslant 30$ & 22806 & 29877 & 41349 & 58131 & 99789 \\
\hline \multicolumn{6}{|l|}{ Pacific } \\
\hline $0-4$ & 1065 & 1818 & 2841 & 5274 & 15288 \\
\hline $5-9$ & 1227 & 1800 & 2727 & 4941 & 14442 \\
\hline $10-14$ & 1227 & 1770 & 2571 & 4881 & 14748 \\
\hline $15-19$ & 1116 & 1803 & 2631 & 5028 & 14169 \\
\hline $20-24$ & 933 & 1545 & 2394 & 4569 & 12408 \\
\hline $25-29$ & 744 & 1308 & 2130 & 3726 & 9204 \\
\hline$\geqslant 30$ & 5679 & 8706 & 12789 & 22404 & 56841 \\
\hline \multicolumn{6}{|l|}{ Asian } \\
\hline $0-4$ & 5493 & 6834 & 7182 & 7572 & 5517 \\
\hline $5-9$ & 5277 & 6264 & 6168 & 5919 & 4551 \\
\hline $10-14$ & 5628 & 6477 & 6195 & 5724 & 4296 \\
\hline $15-19$ & 6267 & 7674 & 7374 & 7533 & 6270 \\
\hline $20-24$ & 5874 & 8031 & 8751 & 10959 & 11076 \\
\hline $25-29$ & 6132 & 8994 & 10620 & 12201 & 10071 \\
\hline$\geqslant 30$ & 44778 & 53430 & 52209 & 50049 & 36879 \\
\hline
\end{tabular}

Values given are $n$.

* All individuals able to be mapped to one of our age, prioritized ethnicity and NZDep deprivation strata $(n=4227981)$. Numbers highlighted in grey indicate strata with stratum-specific predicted risk of 1 case/1000 pyar (Table 3).

It is outside our focus here to discuss the ethical and logistical challenges associated with such interventions. However, if we were to design a national ARF prevention or RHD screening programme based on the estimates of ARF risk we have reported in this paper - and we wished to target our intervention to those strata at highest absolute risk, due to resource constraints or similar - then we may choose to target Māori and Pacific Island children aged 10-14 years residing in NZDep deciles 9-10 (Māori adjusted rate: $4 \cdot 4 / 1000$ pyar; Pacific: $6 \cdot 2 / 1000$ pyar). In order to maximize effectiveness, such a programme would need to ensure equitable provision of the intervention to $>40000$ children; and if we were to expand our risk strata to include 5- to 9-year-olds, then $>80000$ children would need to be targeted (Table 4).

We must consider who might be missed if we were to use such a targeted approach. Outside of the two highest-risk strata discussed above, we observed a number of Māori and Pacific age and deprivation strata that carried an ARF risk $>1$ case/1000 pyar (Table 4) - a not insubstantial burden when we consider the rarity of this disease, even in the highest-risk strata. We also note that nearly a third $(30 \%)$ of all ARF cases occurred outside NZDep deciles 9-10, while a third $(33 \%)$ occurred in those aged outside 
the 5-14 years age group (Table 1). Any national effort that focused only on the highest-risk strata - or only on those schools that are in the poorest socioeconomic areas, as in current programmes - must accept that a substantial minority of the at-risk population will not have the opportunity to benefit from the intervention. Moreover, the spread of risk across multiple strata means that interventions that target populations based only on deprivation may include the highest-risk strata, but they will also $(a)$ include groups with very low risk of ARF, such as non-Māori/non-Pacific children; and (b) exclude some groups with moderate risk of ARF, such as Māori and Pacific children living outside high deprivation areas. It is also worth noting that the measure of deprivation used in this study is based on the address at which an individual usually resides - not the address of the school the individual attends, as might be used in school-based interventions. While there is likely to be confluence between the two, they are certainly not synonymous.

Finally, targeting an intervention (such as RHD screening) towards given age/ethnicity/deprivation strata based on peak disease rates might ignore other important contributors to the overall success of the intervention. It may be that the benefits and risks associated with the intervention are not equal across strata: for example, if one of the goals of RHD screening is to identify those with subclinical disease and intervene with prophylaxis (to minimize risk of ARF recurrence), then it is possible that such an intervention will benefit younger children more than older children. There is limited evidence regarding both the magnitude of the benefits and harms and the optimal timing of such an intervention, and thus further work is required in this area.

\section{Strengths and limitations}

This paper is the first to combine those sociodemographic variables that have been previously associated with ARF development, and present adjusted estimates of disease risk by relevant strata. In this way, we have (uniquely) separated the independent effects of age, ethnicity and deprivation on ARF risk where previously these risks have been conflated.

Our estimates are based on variables available from national-level datasets in New Zealand, with the width of categorizations (e.g. age group 5-9 years, rather than individual years) dependent on availability of age-, ethnicity- and deprivation-stratified population count data. Age and deprivation are, in reality, continuous variables that we have categorized in convenient ways that may not reflect natural and policy relevant cut-off points. For example, it would have been useful to be able to employ age groups that reflected differing school environments (primary school aged 5-12 years, secondary aged 13-17 years).

The ARF dataset is influenced by factors causing it to both under- and over-count the true number of cases. ARF is a syndromic diagnosis, and clinically important disease has a wide spectrum of symptoms from mild (even asymptomatic) to severe. Cases can be missed if these individuals: (a) did not seek medical attention for their symptoms, (b) did not have their (likely mild) symptoms recognized as ARF when they did present to healthcare services, or $(c)$ were neither admitted to hospital nor notified to a medical officer of health, despite being diagnosed with ARF. There is evidence that this case under-ascertainment is large: for example, less than half $(41 \%)$ of the 1016 RHD cases aged $<20$ years diagnosed between 1997 and 2010 had previously been admitted to hospital and diagnosed with ARF [19]. This under-count of the true number of ARF cases over our study period would thus make the stratum-specific rates of disease that we have reported here conservative.

There are also factors leading to some over-count of the true number of cases. Evidence from regional registers-which serve as vital patient management tools - suggests that national-level data overestimate the number of diagnosed ARF cases [20, 21]. In this scenario, our dataset would over-count the number of ARF cases that occurred between 2010 and 2013. Along this line, we also note that recent evidence suggests that rates of ARF in New Zealand appear to have reduced in the years 2014 and 2015 [22] - meaning that the stratum-specific rates of disease reported here may have attenuated somewhat since the end of the study period.

Arguments can be made in either direction regarding whether we have accurately counted the true number of ARF cases and whether our stratum-specific estimates of disease risk are still applicable in the face of a seemingly rapid recent change in the epidemiology of this disease. However, it is likely that none of these scenarios would alter the pattern of disease risk across strata: in other words, Māori and Pacific individuals aged 10-14 years residing in NZDep deciles 9-10 would still be at the greatest risk of ARF - only the absolute magnitude of the stratum-specific risk would be altered. 
We must also consider the possibility that at least some of those cases diagnosed in older age groups (e.g. $\geqslant 20$ years; $18 \%$ of all ARF cases 2010-2013; Table 1) are in fact recurrences of cases that occurred earlier in life - cases that were either $(a)$ not diagnosed and reported to the central data repositories employed in the current study, or $(b)$ occurred prior to 1988 (when NHI use became universal for hospitalization records). Such a scenario would result in an overcounting of cases in older age groups - with an associated increase in stratum-specific rates of disease in that age group.

Finally, in congruence with previous descriptions of the ethnic patterning of ARF in New Zealand [5, 6] the current paper used the 'prioritized' approach to define ethnic groups. This approach simplifies the often-complex dynamic of ethnic affiliation by prioritizing some self-identified ethnicities over others: in our case, Māori ethnicity is first priority, followed by Pacific and then Asian, with those identifying as none of these groups designated as non-Māori/ non-Pacific/non-Asian (or 'European/other'). The limitation of this approach in the current context is that while the number of Māori ARF cases will directly reflect the incidence of ARF in all those who identify as Māori, the number of ARF cases in Pacific individuals will tend to be under-counted-since those who identify as both Māori and Pacific will only be counted as Māori ( $n=29$ cases, or $4 \%$ of our cohort). However, this is also true of the source population-i.e. the denominator data, drawn from the 2013 Census-meaning that this under-count does not necessarily lead to an attenuation of the estimated risk of ARF in Pacific peoples, since both the numerator (ARF cases) and denominator (Census data) are affected.

\section{CONCLUSIONS}

The likelihood of developing ARF varies considerably by age, ethnicity and deprivation strata. While risk is greatest in Māori and Pacific children aged 10-14 years residing in the most extreme levels of deprivation, both of these ethnic groups experience significantly elevated risk across a wide age range from 5 to 29 years and across all levels of deprivation. The presence of substantial risk across multiple strata means that interventions that target populations based on deprivation will include the highest-risk strata, but they will also $(a)$ include groups with very low risk of ARF, such as non-Māori/non-Pacific children; and (b) exclude some groups with moderate risk of ARF, such as Māori and Pacific children living outside high deprivation areas.

\section{SUPPLEMENTARY MATERIAL}

For supplementary material accompanying this paper visit http://dx.doi.org/10.1017/S0950268816001291.

\section{ACKNOWLEDGEMENTS}

We thank Rebekah Roos at the Institute of Environmental Science and Research and Chris Lewis from the Ministry of Health for their assistance with data extraction, and the Health Research Council of New Zealand for funding the study (HRC ref. no. 13/965).

\section{DECLARATION OF INTEREST}

None.

\section{REFERENCES}

1. Carapetis JR, et al. The global burden of group A streptococcal diseases. Lancet Infectious Diseases 2005; 5: 685-694.

2. Williamson D, et al. M-protein analysis of Streptococcus pyogenes isolates associated with acute rheumatic fever in New Zealand. Journal of Clinical Microbiology 2015; 53: $3618-3620$.

3. Shaikh N, Leonard E, Martin JM. Prevalence of streptococcal pharyngitis and streptococcal carriage in children: a meta-analysis. Pediatrics 2010; 126: e557-e564.

4. Webb RH, Grant C, Harnden A. Acute rheumatic fever. British Medical Journal (Online) 2015; 351: h3443.

5. Milne RJ, et al. Incidence of acute rheumatic fever in New Zealand children and youth. Journal of Paediatrics and Child Health 2012; 48: 685-691.

6. Jaine R, Baker M, Venugopal K. Epidemiology of acute rheumatic fever in New Zealand 1996-2005. Journal of Paediatrics and Child Health 2008; 44: 564-571.

7. Vlajinac $\mathbf{H}$, et al. Influence of socio-economic and other factors on rheumatic fever occurrence. European Journal of Epidemiology 1991; 7: 702-704.

8. Gurney $\mathbf{J}$, et al. The incidence of acute rheumatic fever in New Zealand, 2010-2013. New Zealand Medical Journal 2015; 128: 65-67.

9. Webb RH, et al. Optimising echocardiographic screening for rheumatic heart disease in New Zealand: not all valve disease is rheumatic. Cardiology in the Young 2011; 21: 436-443.

10. Heart Foundation of New Zealand. New Zealand guidelines for rheumatic fever: diagnosis, management and secondary prevention of acute rheumatic fever and rheumatic heart disease: update, 2014. 
11. Oliver J, Pierse N, Baker M. Estimating rheumatic fever incidence in New Zealand using multiple data sources. Epidemiology and Infection 2015; 43: 167-177.

12. Ministry of Health. National Minimum Dataset Data Mart Dictionary. Wellington: Ministry of Health, 2010.

13. University of Otago. Socioeconomic deprivation indexes: NZDep and NZiDep. Secondary socioeconomic deprivation indexes: NZDep and NZiDep (http://www. otago.ac.nz/wellington/departments/publichealth/research/ hirp/otago020194.html).

14. Ministry of Health. Ethnicity data protocols for the health and disability sector. Wellington: Ministry of Health, 2004.

15. Statistics New Zealand. Area unit: classification and coding process. Secondary area unit: classification and coding process 2015 (http://www.stats.govt.nz/methods/ classifications-and-standards/classification-related-statsstandards/area-unit/classification-and-coding-process.aspx).

16. Statistics New Zealand. Geographic area files. Secondary geographic area files, 2015.

17. Baker MG, et al. Increasing incidence of serious infectious diseases and inequalities in New Zealand: a national epidemiological study. Lancet 2012; 379: 1112-1119.

18. Engel ME, et al. Genetic susceptibility to acute rheumatic fever: a systematic review and meta-analysis of twin studies. PLoS ONE 2011; 6: e25326.

19. Oliver J, Pierse N, Baker M. Improving rheumatic fever surveillance in New Zealand. Wellington, New Zealand Ministry of Health, 2013.

20. Robin A, et al. The epidemiology of acute rheumatic fever in Northland, 2002-2011. New Zealand Medical Journal 2013; 126: 46-52.

21. Siriett V, et al. The epidemiology of rheumatic fever in the Tairawhiti/Gisborne region of New Zealand: 1997-2009. New Zealand Medical Journal 2012; 125: 8-15.

22. Ministry of Health. Progress on the Better Public Services rheumatic fever target. Secondary Progress on the Better Public Services rheumatic fever target 2015 (http://www.health.govt.nz/about-ministry/what-we-do/ strategic-direction/better-public-services/progress-betterpublic-services-rheumatic-fever-target). 